University of Nebraska - Lincoln

DigitalCommons@University of Nebraska - Lincoln

June 2004

\title{
Natural Selection Drives Altitudinal Divergence at the Albumin Locus in Deer Mice, Peromyscus Maniculatus
}

Jay F. Storz

University of Nebraska - Lincoln, jstorz2@unl.edu

Jean M. Dubach

Brookfield Zoo, Brookfield, Illinois

Follow this and additional works at: https://digitalcommons.unl.edu/bioscistorz

Part of the Genetics and Genomics Commons

Storz, Jay F. and Dubach, Jean M., "Natural Selection Drives Altitudinal Divergence at the Albumin Locus in Deer Mice, Peromyscus Maniculatus" (2004). Jay F. Storz Publications. 8.

https://digitalcommons.unl.edu/bioscistorz/8

This Article is brought to you for free and open access by the Papers in the Biological Sciences at DigitalCommons@University of Nebraska - Lincoln. It has been accepted for inclusion in Jay F. Storz Publications by an authorized administrator of DigitalCommons@University of Nebraska - Lincoln. 


\title{
Natural Selection Drives Altitudinal Divergence at the Albumin Locus in Deer Mice, Peromyscus Maniculatus
}

\author{
Jay F. Storz ${ }^{*} \dagger$ and Jean M. Dubach ${ }^{\S}$ \\ * Department of Ecology and Evolutionary Biology, University of Arizona, Biosciences West, Tucson, Arizona 85721 \\ $\S$ Department of Conservation Biology, Brookfield Zoo, Brookfield, Illinois 60513 \\ ${ }^{\dagger}$ Present address: Department of Biology, San Francisco State University, 1600 Holloway Avenue, San Francisco, California 94132
}

\begin{abstract}
In populations that are distributed across steep environmental gradients, the potential for local adaptation is largely determined by the spatial scale of fitness variation relative to dispersal distance. Since altitudinal gradients are generally characterized by dramatic ecological transitions over relatively short linear distances, adaptive divergence across such gradients will typically require especially strong selection to counterbalance the homogenizing effect of gene flow. Here we report the results of a study that was designed to test for evidence of adaptive divergence across an altitudinal gradient in a natural population of deer mice, Peromyscus maniculatus. We conducted a multilocus survey of allozyme variation across a steep altitudinal gradient in the southern Rocky Mountains that spanned several distinct biomes, from prairie grassland to alpine tundra. As a control for the effects of altitude, we also surveyed the same loci in mice sampled along a latitudinal transect through the prairie grassland that ran perpendicular to the east-west altitudinal transect. We used a coalescent-based simulation model to identify loci that deviated from neutral expectations, and we then assessed whether locus-specific patterns of variation were nonrandom with respect to altitude. Results indicated that the albumin locus (Alb) reflects a history of diversifying selection across the altitudinal gradient. This conclusion is supported by two main lines of evidence: (1) Alb was characterized by levels of divergence across the altitudinal transect that exceeded neutral expectations in two consecutive years of sampling (in contrast to the spatial pattern of variation across the latitudinal transect), and (2) levels of divergence at the Alb locus exhibited a positive association with altitudinal distance in both years (in contrast to the pattern observed at unlinked loci). We conclude that clinal variation at the Alb locus reflects a balance between gene flow and diversifying selection that results from elevational changes in fitness rankings among alternative genotypes.
\end{abstract}

Keywords: adaptation, allozymes, clinal variation, coalescent, elevational gradient, interlocus contrasts, mice

Can diversifying selection drive adaptive differentiation in the face of countervailing gene flow? This question is of fundamental importance to our understanding of local adaptation and the determinants of geographic variation (Endler 1977). When a population is distributed across a steep environmental gradient, clinal variation in allele frequencies can be maintained by the interplay between diversifying selection and gene flow (Haldane 1948; Slatkin 1973; Endler 1977). In natural populations, clines across latitudinal and altitudinal gradients are often attributed to this type of selection-migration balance (Endler 1986). Indeed, latitudinal clines in allele frequencies provide the basis for some of the most compelling case studies of how positive selection shapes patterns of adaptive genetic variation in natural populations (e.g., Powers and Place 1978; Oakeshott et al. 1981, 1982; Berry and Kreitman 1993; Schmidt et al. 2000; Verrelli and Eanes 2001; Duvernell et al. 2003). Altitudinal clines in allele frequencies are of particular interest since similar gradients of environmental variation will typically span a much shorter physical distance relative to the spatial scale of dispersal. Thus, relative to latitudinal clines, the stable maintenance of altitudinal clines will typically require especially strong selection to counterbalance the homogenizing effect of gene flow.

In the absence of barriers to dispersal, steep clines in allele frequencies may often reflect a history of diversifying selection. However, clines can also be produced by the interplay between drift and spatially restricted gene flow (isolation by distance) or by admixture between previously isolated populations that have come into secondary contact. One means of distinguishing between adaptive versus nonadaptive causes of clinal variation at specific genes is to compare relative levels of spatial differentiation at multiple, unlinked loci. The premise of this approach is that the effects of selection are generally locus-specific, whereas demographic processes are expected to have relatively uniform effects across the entire genome (Cavalli-Sforza 1966; Lewontin and Krakauer 1973).

The purpose of this study was to test for evidence of adaptive divergence across an altitudinal gradient in a natural population of deer mice, Peromyscus maniculatus. Specifically, we analyzed patterns of allozyme variation across a steep altitudinal gradient in the southern Rocky Mountains that spanned several distinct biomes, from prairie grassland to alpine tundra. Peromyscus maniculatus has the broadest altitudinal distribution of any North American mammal and thus represents an ideal taxon for investigating the process of adaptive divergence across environmental gradients.

A previous analysis identified the albumin locus $(A l b)$ as a candidate gene for local adaptation in four different species of Peromyscus (Storz and Nachman 2003). Evidence for diversifying selection on Alb polymorphism was particularly strong in P. maniculatus from Colorado. The P. maniculatus data set analyzed by Storz and Nachman (2003) was based on population samples from a range of different altitudes, suggesting that selection may have been mediated by some factor or combination of factors related to altitude. To investigate this pattern in more detail, and to test for evidence of adaptive divergence across the altitudinal gradient, we augmented the dataset analyzed by Storz and Nachman (2003) by adding data from mice that were collected from three localities at lower altitudes dur- 
ing the same year. This expanded sample allows us to examine spatial patterns of variation over a more continuous range of altitudes. We also surveyed allozyme variation in mice sampled from the same altitudinal transect in a second consecutive year (to assess the temporal consistency of the spatial pattern) and in mice sampled from a latitudinal transect through the prairie grassland (to control for the effects of altitude on patterns of differentiation). If Alb polymorphism is in fact subject to diversifying selection across the altitudinal gradient, levels of divergence at the Alb locus should exceed neutral expectations in temporal replicates of the same altitudinal transect (but not in the latitudinal transect), and allele frequencies should exhibit a consistent, nonrandom association with altitude (in contrast to the patterns of variation at unlinked loci).

\section{Methods}

\section{Sampling the Altitudinal Transect}

A total of 614 P. maniculatus was live-trapped from 11 localities in Colorado during each of two consecutive years. Sampling localities were distributed along an altitudinal transect that spanned approximately $3050 \mathrm{~m}$ of vertical relief over a linear distance of $500 \mathrm{~km}$ (Figure 1A). Sampling localities were spaced along the transect at intervals of about $300 \mathrm{~m}$ elevation, except for site 6 (Black Hawk, $2697 \mathrm{~m}$ ) and site 7 (Echo Lake, $3200 \mathrm{~m}$ ) which were separated by an altitudinal distance of 503 m. Also, site 8 (Kingston Peak, 3475 m) and site 9 (Mt. Evans [I], $3505 \mathrm{~m}$ ) were sampled at approximately the same elevation. Mice were sampled in early summer, prior to the onset of summer breeding activity. Only overwintering adults were used in the analysis of allozyme variation. Populations of P. maniculatus are characterized by extremely high rates of turnover (e.g., O'Farrell 1978), so it is unlikely that our samples from consecutive years contained mice that were born in the same generation.

\section{Sampling the Latitudinal Transect}

In a similar fashion, a total of 112 mice were live-trapped from five localities along a latitudinal transect that ran parallel to the Front Range of the Colorado Rockies (Figure 1B). The latitudinal transect was intended as a control for the effects of altitude on the spatial patterning of allozyme variation. Sampling localities were all situated in prairie grassland habitat at roughly the same altitude (about 1960-1980 m). The latitudinal transect spanned $200 \mathrm{~km}$ and intersected the lower end of the eastwest altitudinal transect at Golden, the only locality that was common to both transects. Samples from the latitudinal transect were collected during the same season as the year 1 altitudinal samples, so the same sample of mice from Golden was used in both analyses.

\section{Allozyme Analysis}

Liver, kidney, blood plasma, or hemolysate preparations were subjected to electrophoretic analysis following the procedures of Selander et al. (1971). Horizontal starch-gel electrophoresis was used to measure variation at a total of 18 enzymatic and non-enzymatic proteins (all data were collected by JMD).
The enzymes (and associated Enzyme Commission numbers) included alcohol dehydrogenase (Adh-1; 1.1.1.1), aspartate aminotransferase (Aat-1 and Aat-2; 2.6.1.1), esterase (Est-4; 3.1.1.1), glucose-6-phosphate dehydrogenase (G6pd; 1.1.1.49), glycerol-3-phosphate dehydrogenase ( $\alpha-G p d$; 1.1.1.8), isocitrate dehydrogenase $(I d h ; 1.1 .1 .42)$, lactate dehydrogenase $(L d h-1$ and $L d h-2 ; 1.1 .1 .27)$, malate dehydrogenase (Mdh-1 and $M d h$ 2; 1.1.1.37), phosphoglucomutase (Pgm-1; 2.7.5.1), 6-phosphogluconate dehydrogenase (6-Pgd; 1.1.1.44), sorbitol dehydrogenase (Sdh; 1.1.1.14), and superoxide dismutase (Sod; 1.15.1.1). The nonenzymatic proteins included albumin (Alb), hemoglobin $(H b)$, and transferrin $(T r f)$. For all of the allozyme loci included in this survey of $P$. maniculatus, codominant segregation of electrophoretic variants has been confirmed by breeding experiments (e.g., Baccus et al. 1980; Nadeau and Baccus 1981; Aquadro and Avise 1982; Dawson 1982; Dawson et al. 1983). A subset of the year 1 data (localities 4-10 from the altitudinal transect) was included in a previous analysis by Storz and Nachman (2003).

\section{Allelic and Genotypic Disequilibria}

We used a randomization procedure to test for evidence of linkage disequilibrium between each pairwise combination of loci. Genotypes at pairs of loci were associated at random and the log-likelihood $G$-statistic was calculated for each randomized data set. $P$-values for each pairwise combination of loci were calculated as the proportion of randomized datasets with values of the $G$-statistic that were equal to or greater than the observed value. For each year, null distributions of the $G$-statistic were generated from $[20 L(L-1)] / 2$ randomizations where $L$ is the number of polymorphic loci. In a similar fashion, we used a randomization procedure to test for departures from HardyWeinberg genotypic proportions using Weir and Cockerham's (1984) estimators of the inbreeding coefficients: $f\left(=F_{\text {IS }}\right)$ and $F\left(=F_{\text {IT }}\right)$. Null distributions were generated from 10,000 randomizations of alleles among individuals within samples (for $F_{\text {IS }}$ ) and among individuals from the total set of samples (for $\left.F_{\text {IT }}\right)$. Ninety-five percent confidence intervals were obtained by bootstrapping over loci. The program FSTAT version 2.9.3 (updated from Goudet 1995) was used for all calculations.

\section{Testing for Evidence of Selection}

Previous efforts to develop an $F_{\mathrm{ST}}$-based neutrality test (e.g., Lewontin and Krakauer 1973) were criticized on the grounds that parametric expectations for the interlocus variance in $F_{\mathrm{ST}}$ did not account for patterns of migration or population history that could induce a correlation in allele frequencies among subpopulations (Nei and Maruyama 1975; Robertson 1975a,b). An alternative approach is to use a coalescent simulation model to generate null distributions of $F_{\mathrm{ST}}$ (conditional on heterozygosity) under a neutral model of population structure (Beaumont and Nichols 1996; see also McDonald 1994; Vitalis et al. 2001; Porter 2003).

Considering each of the three datasets separately (the latitudinal transect and two replicates of the altitudinal transect), we obtained single-locus estimates of $F_{\mathrm{ST}}$ using the $\beta$ statistic of Cockerham and Weir (1993): 

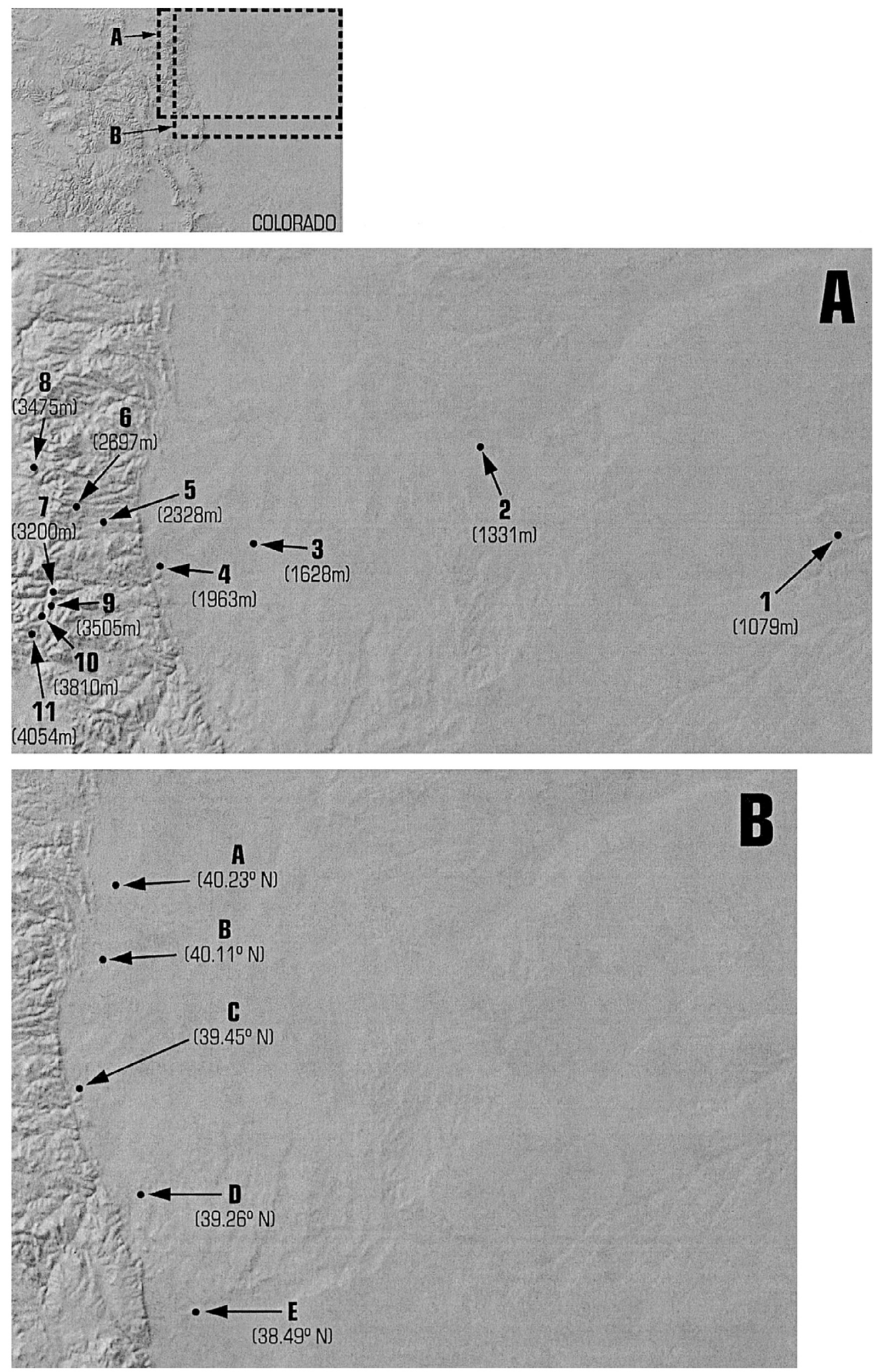

Figure 1. Map of northeastern Colorado showing localities where deer mice were sampled for the analysis of allozyme variation. (A) Map of the altitudinal transect. Names of sampling localities, elevation, and sample size (no. of mice sampled in year 1/year 2) are as follows: 1, Wray, 1079 m (28/23); 2, Fort Morgan, 1331 m (49/45); 3, Northglenn, 1628 m (52/43); 4, Golden, 1963 m (42/34); 5, Golden Gate State Park, 2328 m (0/29); 6, Black Hawk, 2697 m (6/22); 7, Echo Lake, 3200 m (14/14); 8, Kingston Peak, 3475 m (16/ 23); 9, Mt. Evans (I), 3505 m (40/30); 10, Mt. Evans (II), 3810 m (30/23); and 11, Mt. Evans (III), 4054 m (31/20). (B) Map of the latitudinal transect. Names of sampling localities, degrees north latitude, and sample size (no. of mice) are as follows: A, Carter Lake, $40.23^{\circ} \mathrm{N}$ (17); B, Longmont, $40.11^{\circ} \mathrm{N}$ (14); C, Golden, $39.45^{\circ} \mathrm{N}$ (42); D, Sedalia, 39.26 ${ }^{\circ} \mathrm{N}(14)$; E, Colorado Springs, $38.49^{\circ} \mathrm{N}(25)$. 


$$
\beta=\left(F_{0}-F_{1}\right) /\left(1-F_{1}\right)
$$

where $1-F_{1}$ is the average probability of identity-by-descent for two alleles sampled randomly from separate subpopulations, and $1-F_{0}$ is the average probability of identity-by-descent for two alleles sampled randomly from the same subpopulation. We estimated heterozygosity, $H$, as $1-F_{1}$. For each data set, we calculated the expected $F_{\mathrm{ST}}$ as the among-locus average weighted by $H$ (Weir and Cockerham 1984). We then tested for evidence of selection by comparing observed $F_{\text {ST }}$ values to a null distribution generated by a coalescent-based simulation model. Specifically, we used the structured coalescent model of Beaumont and Nichols (1996) to generate a null distribution of $F_{\mathrm{ST}}$ conditional on $H$. We performed coalescent simulations using a symmetrical 100 -island model of population structure and two different mutation models: the infinite alleles model (IAM; Kimura and Crow 1964) and the stepwise mutation model (SMM; Ohta and Kimura 1973). Sample sizes were set equal to the median of actual sample sizes in each of the three datasets. After generating a heterozygosity value uniformly on $(0,1)$, values of $N_{e} \mu$ (the mutation rate scaled to effective population size) were derived from the following relationships:

$$
H=4 N_{e} \mu /\left(1+4 N_{e} \mu\right)
$$

for the IAM (Kimura and Crow 1964), and

$$
H=1-1 /\left(8 N_{e} \mu\right)^{0.5}
$$

for the SMM (Ohta and Kimura 1973). Coalescent simulations were used to generate a total of 50,000 paired values of $F_{\mathrm{ST}}$ and $H$. To compute the conditional probability density of $F_{\mathrm{ST}}$, we used a computer program written by M. A. Beaumont (available at http://www.rubic.rdg.ac.uk/ mab/software.html).

To assess the statistical significance of departures from the neutral expectation, two-tailed probabilities were expressed as the proportion of 50,000 simulated values that were more extreme than the observed values. For each dataset, the $\alpha$-level was Bonferroni-adjusted for multiple comparisons $(0.05 /$ number of loci).

\section{Testing for Evidence of Adaptive Divergence across the Altitudinal Gradient}

After identifying loci that deviated from neutral expectations, we tested for nonrandom associations with altitude. For each pairwise combination of sampling localities, arcsine-transformed values of $F_{\mathrm{ST}}^{1 / 2}$ were calculated separately for each putatively selected locus $\left(F_{\mathrm{ST}(\text { selected) }}\right)$ and the remaining putatively neutral loci $\left(F_{\mathrm{ST}(\text { neutral }}\right)$. Because oxygen tension, ambient temperature, and climatic variability covary with altitude, pairwise difference in altitude can be considered as a proxy measure for overall environmental dissimilarity between sampling localities. Altitudinal distances were computed as $\left(\left(x_{i}-x_{j}\right)^{2}\right)^{1 / 2}$, where $x_{i}$ and $x_{j}$ represent altitudes for the $i$ th and $j$ th localities.
If altitudinal clines in allele frequencies are simply attributable to stochastic differentiation via drift, a positive association between $F_{\mathrm{ST} \text { (selected) }}$ and altitudinal distance would not be expected to remain statistically significant after controlling for $F_{\text {ST(neutral) }}$. The null hypothesis of neutral divergence can be tested using a multiple regression model (Storz 2002). A significant partial regression of $F_{\mathrm{ST}(\text { selected) }}$ on altitudinal distance would indicate that migration-drift equilibrium is not a sufficient explanation for the observed pattern of differentiation. We used partial Mantel tests to assess the association between $F_{\mathrm{ST}(\text { selected) }}$ and altitudinal distance. These tests involve simultaneous comparisons of one dependent variable matrix and multiple independent variable matrices. For the partial Mantel tests, a matrix of $F_{\mathrm{ST}(\text { selected) }}(\mathrm{S})$ was related to a matrix of $F_{\mathrm{ST}(\text { neutral }}(\mathbf{N})$ and a matrix of pairwise measures of altitudinal distance (A). For the three respective matrices, let $s_{i j}$, $n_{i j}$, and $a_{i j}$ denote distances between localities $i$ and $j$. We then fit the following regression equation:

$$
s_{i j}=c_{v(i) v(j)}+\beta_{1} n_{i j}+\beta_{2} a_{i j},
$$

where $\beta_{2}$ measures the association between $s_{i j}$ and $a_{i j}$ while controlling for the effects of $n_{i j}$, and $c_{v(i) v(j)}$ is a nuisance parameter that depends on the group identities of the localities under consideration. Specifically, $v(i)$ denotes the group identity of locality $i$, and $c_{r s}$ takes into account the overall difference in $F_{\mathrm{ST}(\text { selected) }}$ between localities belonging to groups $r$ and $s$.

To assess the statistical significance of the tests, we used a matrix randomization procedure in which permutations of the response matrix were restricted to two spatially defined groups of localities along the altitudinal transect: prairie grassland localities (1-4) and montane localities (5-11; Figure 1A). After constructing a null distribution of regression coefficients by iteratively randomizing $s_{i j}$ within the high- and low-altitude groups, probability values for the null hypothesis of no association were then expressed as the proportion of 10,000 matrix randomizations that yielded $t$-values greater than or equal to the observed value.

This restricted randomization procedure permits an assessment of the association between pairwise measures of $F_{\mathrm{ST}(\text { selected) }}$ and altitudinal distance after allowing for large-scale differences between high- and low-altitude groups of localities, as well as small-scale differences within groups. Since restricted randomizations account for the spatial structure of the data, this procedure should account for any residual source of spatial autocorrelation that is not removed by including the matrix of $F_{\mathrm{ST}(\text { neutral) }}$ as an independent variable in the regression model (Sokal et al. 1989, 1990; Manly 1997).

We tested for evidence of spatial autocorrelation in the residuals of the fitted equation as follows. For each pair of localities we measured the difference in the residuals from separate regressions of the response matrix $\mathbf{S}$ on the two independent variable matrices. We calculated the residuals as the differences in observed values of $F_{\mathrm{ST} \text { (selected) }}$ between localities and the differences predicted by fitting equation (4). Residual differences between localities $i$ and $j$ were calculated as 


$$
d_{i j}=\sqrt{\sum_{\substack{k=1 \\ k \neq i, j}}^{n} \frac{\left(r_{i k}-r_{j k}\right)^{2}}{(n-2)}},
$$

where $n$ is the number of localities and $r_{i k}$ is the residual difference between localities $i$ and $k$. We then assessed whether these pairwise residual differences were positively correlated with pairwise measures of $F_{\mathrm{ST} \text { (neutral) }}$.

To determine whether the altitudinal and latitudinal transects were characterized by any detectable difference in the spatial scale of gene flow, we tested for evidence of isolation by distance (Wright 1943) by performing a regression of pairwise $F_{\text {ST(neutral) }}$ against $\ln$-transformed measures of pairwise geographic distance for each transect. We tested for significance of the regression coefficients using matrix randomizations as described above.

\section{Results}

Three of the 18 loci analyzed in P. maniculatus from the altitudinal transect were monomorphic in both years (Mdh-2, G6pd, and Sod) and $L d h-2$ was monomorphic in samples from year 1. For the remaining loci, expected heterozygosity averaged 0.206 in year 1 ( $n=308$ mice) and 0.230 in year $2(n=306$ mice; Table 1). Two additional loci were monomorphic in mice sampled from the latitudinal transect (Aat-2 and $L d h-1$ ) and expected heterozygosity at the remaining 13 loci averaged $0.201(n=112$ mice; Table 1).

\section{Tests of Allelic and Genotypic Disequilibria}

Randomization tests revealed six significant cases of pairwise linkage disequilibrium in the year 1 altitudinal transect $(L d h$ $2 \times$ Adh, $6-P g d \times \alpha-G p d$, Est-4 $\times$ Adh, Est-4 $\times$ Pgm, Est-4 $\times$ $\operatorname{Trf}$, and $\alpha-G p d \times A d h$ ), four cases in the year 2 altitudinal transect $(L d h-1 \times H b, I d h-1 \times M d h-1$, Est-4 $\times$ Aat-2, and Aat-1 $\times$ Aat-2), and three cases in the latitudinal transect (Aat-1 $\times$ Pgm, $S d h \times \operatorname{Trf}$, and $\mathrm{Pgm} \times \mathrm{Alb}$ ). However, no pairwise associations were statistically significant after correcting for multiple comparisons. The same was true on a within-locality basis.

Both years of sampling across the altitudinal transect were characterized by a slight deficit of heterozygotes within localities (mean $F_{\text {IS(year 1) }}=0.127,95 \%$ CI $=0.036$ to 0.210 ; mean $\left.F_{\text {IS(year 2) }}=0.059,95 \% \mathrm{CI}=-0.022-0.166\right)$ and among localities (mean $F_{\text {IT(year 1) }}=0.171,95 \%$ CI $=0.093-0.247$; mean $F_{\text {IT(year 2) }}=0.097,95 \% \mathrm{CI}=0.019-0.202$ ). Out of 150 locus $\times$ locality combinations in year 1 , randomization tests revealed 12 cases of a within-sample heterozygote deficit (6-Pgd in localities 2, 3, 4, 9, and 10; Est-4 in locality 1; Aat-1 in locality 9; and Adh in localities 1, 2, 4, 9, and 11) and one case of a heterozygote excess (Aat-1 in locality 2 ). Out of 154 locus $\times$ locality combinations in year 2, randomization tests revealed eight cases of a heterozygote deficit (Aat-1 in locality 6; Alb in locality 11 ; and $H b$ in localities 2, 5, 8, 9, 10, and 11) and one case of a heterozygote excess (6-Pgd in locality 11). However, none of the single-locus $F_{\text {IS }}$ values remained significant after correcting for multiple comparisons. Sampling across the latitudinal
Table 1. Patterns of variation at 15 allozyme loci used in the genetic analysis of deer mice from Colorado. In each row: altitudinal transect, year 1, top; altitudinal transect, year 2, middle; latitudinal transect, bottom. Samples sizes for all localities are given in the legend for Figure 1. $N_{\mathrm{A}}=$ number of alleles.

\begin{tabular}{|c|c|c|c|c|c|}
\hline & $N_{\mathrm{A}}$ & $H$ & $F_{\mathrm{IS}}$ & $F_{\text {IT }}$ & $F_{\mathrm{ST}}$ \\
\hline \multirow{3}{*}{$A l b$} & 3 & 0.331 & 0.000 & 0.166 & 0.218 \\
\hline & 3 & 0.378 & 0.022 & 0.115 & 0.107 \\
\hline & 2 & 0.306 & -0.005 & 0.012 & 0.023 \\
\hline \multirow[t]{3}{*}{ Adh-1 } & 2 & 0.160 & 0.545 & 0.558 & 0.042 \\
\hline & 3 & 0.380 & 0.136 & 0.144 & 0.015 \\
\hline & 3 & 0.092 & 0.171 & 0.170 & 0.007 \\
\hline \multirow[t]{3}{*}{ Aat-1 } & 3 & 0.503 & 0.027 & 0.043 & 0.006 \\
\hline & 3 & 0.479 & -0.066 & -0.023 & 0.040 \\
\hline & 2 & 0.475 & 0.001 & -0.020 & -0.022 \\
\hline \multirow[t]{3}{*}{ Aat-2 } & 2 & 0.006 & 0.002 & -0.002 & -0.002 \\
\hline & 3 & 0.019 & -0.031 & -0.002 & 0.024 \\
\hline & 1 & 0.000 & - & - & - \\
\hline \multirow[t]{3}{*}{ Est-4 } & 3 & 0.483 & 0.064 & 0.124 & 0.071 \\
\hline & 4 & 0.567 & 0.055 & 0.082 & 0.028 \\
\hline & 3 & 0.503 & 0.129 & 0.159 & 0.039 \\
\hline \multirow[t]{3}{*}{$\alpha-G p d$} & 3 & 0.031 & -0.035 & -0.003 & 0.032 \\
\hline & 3 & 0.020 & -0.004 & -0.006 & -0.002 \\
\hline & 3 & 0.026 & -0.014 & 0.004 & -0.004 \\
\hline \multirow[t]{3}{*}{$\mathrm{Hb}$} & 2 & 0.504 & 0.182 & 0.234 & 0.096 \\
\hline & 2 & 0.504 & 0.333 & 0.363 & 0.065 \\
\hline & 2 & 0.492 & 0.114 & 0.144 & 0.043 \\
\hline \multirow[t]{3}{*}{$I d h$} & 3 & 0.073 & -0.025 & -0.022 & 0.001 \\
\hline & 3 & 0.050 & -0.025 & -0.015 & 0.007 \\
\hline & 3 & 0.033 & -0.002 & -0.002 & 0.012 \\
\hline \multirow[t]{3}{*}{$L d h-1$} & 2 & 0.003 & 0.004 & -0.001 & 0.000 \\
\hline & 3 & 0.011 & -0.018 & -0.001 & 0.015 \\
\hline & 1 & 0.000 & - & - & - \\
\hline \multirow[t]{3}{*}{$L d h-2$} & 3 & 0.019 & -0.003 & -0.005 & 0.014 \\
\hline & 1 & 0.000 & - & - & - \\
\hline & 3 & 0.021 & -0.002 & -0.005 & 0.002 \\
\hline \multirow[t]{3}{*}{$M d h-1$} & 2 & 0.002 & 0.004 & -0.001 & 0.000 \\
\hline & 3 & 0.012 & -0.004 & -0.004 & 0.001 \\
\hline & 3 & 0.033 & -0.019 & -0.002 & 0.012 \\
\hline \multirow[t]{3}{*}{ Pgm-1 } & 4 & 0.034 & -0.018 & -0.009 & 0.010 \\
\hline & 3 & 0.088 & -0.042 & -0.037 & 0.006 \\
\hline & 3 & 0.073 & -0.067 & -0.010 & 0.053 \\
\hline \multirow[t]{3}{*}{ 6-Pgd } & 4 & 0.643 & 0.216 & 0.233 & 0.016 \\
\hline & 5 & 0.694 & -0.021 & 0.010 & 0.039 \\
\hline & 4 & 0.683 & 0.215 & 0.226 & 0.015 \\
\hline \multirow[t]{3}{*}{$S d h$} & 2 & 0.048 & -0.054 & -0.015 & 0.047 \\
\hline & 3 & 0.054 & 0.108 & 0.117 & 0.007 \\
\hline & 2 & 0.033 & -0.025 & -0.016 & 0.011 \\
\hline \multirow[t]{3}{*}{$\operatorname{Trf}$} & 3 & 0.246 & 0.065 & 0.093 & 0.008 \\
\hline & 3 & 0.196 & -0.047 & 0.044 & 0.068 \\
\hline & 3 & 0.249 & 0.180 & 0.181 & 0.016 \\
\hline
\end{tabular}

transect also revealed a slight deficit of heterozygotes within localities (mean $F_{\text {IS }}=0.109,95 \% \mathrm{CI}=0.032-0.164$ ) and among localities (mean $F_{\text {IT }}=0.123,95 \% \mathrm{CI}=0.037-0.178$ ). As in the case with samples from the altitudinal transect, no within-locality departures from Hardy-Weinberg proportions remained significant after correcting for multiple comparisons.

\section{Tests for Evidence of Selection}

Analysis of the year 1 data revealed an extremely low degree of genetic subdivision across the altitudinal gradient (weighted mean $F_{\mathrm{ST}}=0.059, n=15$ loci). The expected distribution of $F_{\mathrm{ST}}$ as a function of $H$ gave a poor fit to the data under both mutation models, since more points fell below the 0.025 quantile than expected by chance (Figure 2A). The conditional probability distributions were virtually identical under both mutation 

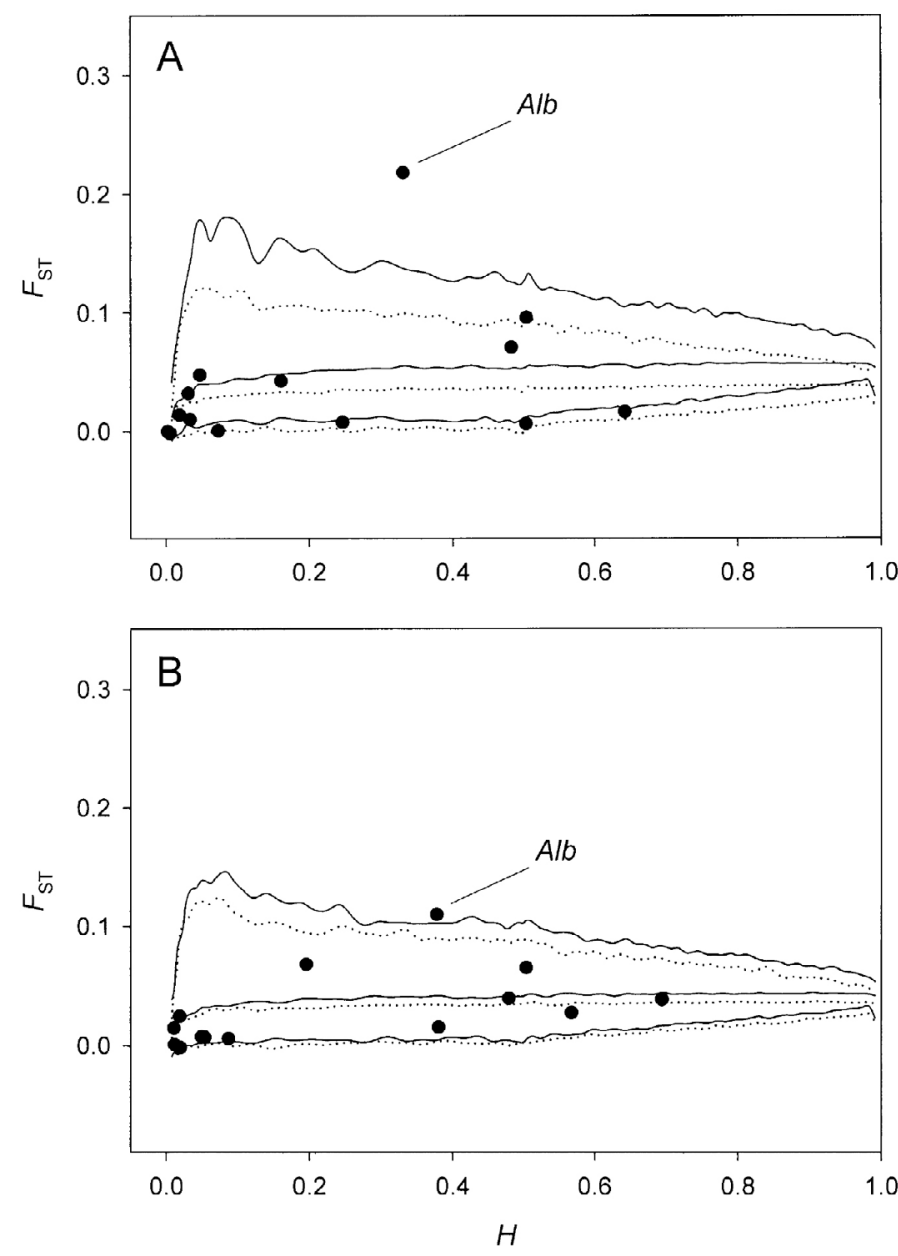

Figure 2. Estimated $F_{\mathrm{ST}}$ values from allozyme loci plotted as a function of heterozygosity in P. maniculatus from the altitudinal transect. Results are shown for (A) year 1 and (B) year 2. Lines denote the 0.975, 0.500, and 0.025 quantiles of the conditional distribution. Simulation-based expectations were obtained under the infinite alleles model of mutation. Dotted lines denote quantiles of the distribution that were recalculated after removing Alb.

models, consistent with the simulation results of Beaumont and Nichols (1996) and Storz and Nachman (2003). Only results obtained under the IAM will be presented in the remainder of the paper. The poor fit to the data was attributable to a single locus (Alb) that was characterized by an unusually high level of differentiation relative to the remaining 14 loci (Table 1). The departure from neutral expectations at the Alb locus remained statistically significant after correcting for multiple comparisons $(P$ $=0.0018$; Bonferroni-adjusted $\alpha$-level $=0.0033)$. The weighted mean $F_{\mathrm{ST}}$ was reduced to 0.039 after removing this outlier, and we used this new average to recalculate the expected neutral distribution. The $F_{\mathrm{ST}}$ values for almost all remaining loci fell within the 0.025 and 0.975 quantiles of the recalculated distribution (Figure 2A).

We observed a similar pattern in the year 2 data. Again, the data revealed an extremely low degree of genetic subdivision across the altitudinal gradient (weighted mean $F_{\mathrm{ST}}=0.045, n=$ 14 loci). The majority of loci exhibited a remarkably close fit to the expectations of the neutral model (Figure 2B). The only ex-

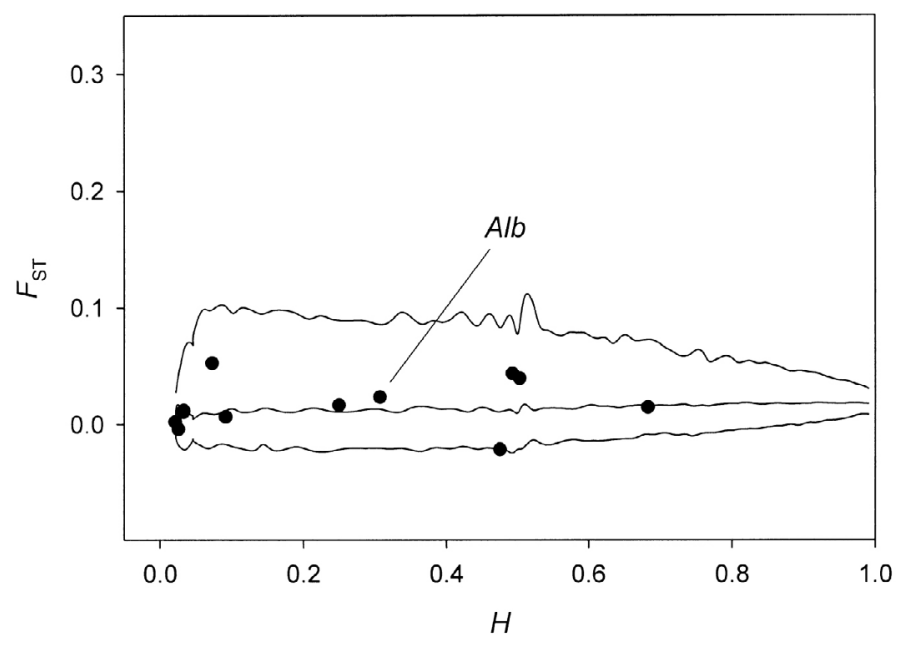

Figure 3. Estimated $F_{\mathrm{ST}}$ values from allozyme loci plotted as a function of heterozygosity in Peromyscus maniculatus from the latitudinal transect. Lines denote the $0.975,0.500$, and 0.025 quantiles of the conditional distribution. Simulation-based expectations were obtained under the infinite alleles model of mutation.

ception was $A l b$, which was again characterized by the highest $F_{\text {ST }}$ value of all loci (Table 1). The deviation from the neutral expectation did not remain statistically significant after correcting for multiple comparisons $(P=0.0293$; Bonferroni-adjusted $\alpha$-level $=0.0036$ ). The weighted mean $F_{\mathrm{ST}}$ was reduced to 0.038 after removing this outlier, but this adjustment did not dramatically alter the fit of the data to the conditional distribution (Figure 2B).

As with the altitudinal transects, data from the latitudinal transect revealed an extremely low degree of genetic subdivision (weighted mean $F_{\mathrm{ST}}=0.019, n=13$ loci). In contrast to the altitudinal patterns of variation, data from the latitudinal transect revealed no evidence of selection on Alb polymorphism (Figure 3). All loci closely conformed to expectations of the neutral model.

\section{Tests of Adaptive Divergence across the Altitudinal Gradient}

Having determined that the Alb locus exhibits a higher-thanexpected level of divergence across the altitudinal gradient, we then performed partial Mantel tests to assess whether levels of divergence exhibited a nonrandom pattern with respect to altitude. In both years, $F_{\mathrm{ST}}$ for $A l b\left(F_{\mathrm{ST}(A l b)}\right)$ exhibited a stronger positive association with altitudinal distance than did the weighted mean $F_{\mathrm{ST}}$ for all remaining neutral loci ( $F_{\mathrm{ST}(\text { neutral)}}$; Figure 4). The partial Mantel test with restricted randomizations revealed a statistically significant association between $F_{\mathrm{ST}(\mathrm{Alb})}$ and altitudinal distance in both years. In other words, the increase in pairwise $F_{\mathrm{ST}(\mathrm{Alb})}$ as a positive function of pairwise altitudinal distance remained significant even when the effects of neutral divergence (as measured by $F_{\text {ST(neutral) }}$ ) were partialled out. In both years, regression coefficients for both $F_{\mathrm{ST} \text { (neutral) }}$ and altitudinal distance were significantly greater than zero, and $F$-values for the extra sum of squares accounted for by adding each variable into the equation were also significant at an $\alpha$ level of 0.05 (Table 2). 

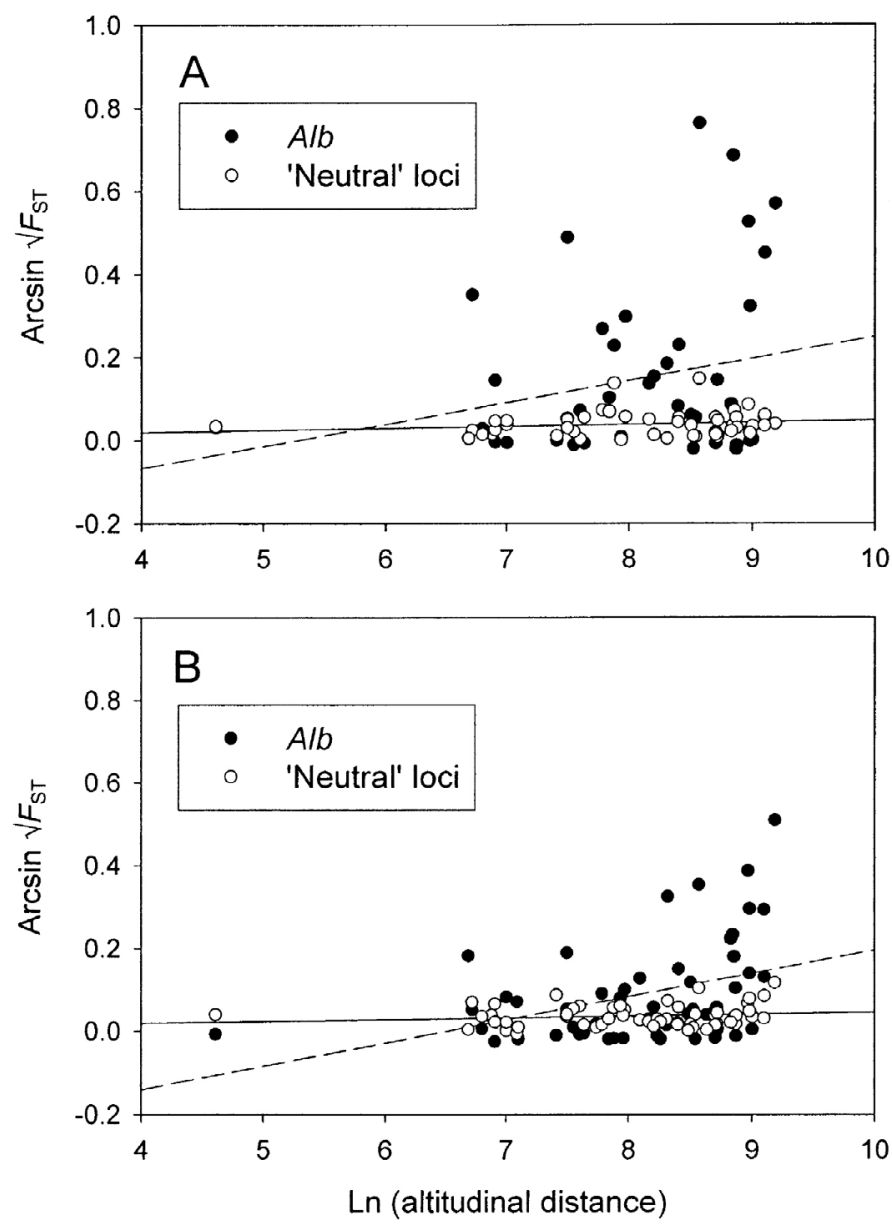

Figure 4. Linear regression of arcsin $\left(F_{\mathrm{ST}}\right)^{\frac{1}{2}}$ against $\ln$ (altitudinal distance) for each pairwise combination of sampling localities in (A) year 1 and (B) year 2. The solid line denotes the linear regression line for transformed values of $F_{\mathrm{ST}(\text { neutral) }}$ versus altitudinal distance, and the dashed line denotes the linear regression line for transformed values of $F_{\mathrm{ST}(A l b)}$ versus altitudinal distance.

To test for evidence of autocorrelation in the residuals of the fitted equation, we performed a regression of residual differences between localities $\left(d_{i j}\right)$ against pairwise values of $F_{\mathrm{ST} \text { (neutral) }}\left(n_{i j}\right)$. The regressions gave the fitted equations $d_{i j}=$ $0.206+0.171 n_{i j}$ for year 1 , and $d_{i j}=0.135-0.103 n_{i j}$ for year 2. Mantel tests with unrestricted randomizations showed that the regression coefficients were not significantly greater than zero ( $P=0.583$ and 0.598 in years 1 and 2, respectively), in- dicating that the positive associations between $F_{\mathrm{ST}(A l b)}$ and altitudinal distance cannot be explained as an artifact of spatial autocorrelation.

The striking pattern of spatial differentiation at the Alb locus reflects a steep altitudinal cline in $A l b$ allele frequencies (Figure 5A,B). Averaging across both years, the $b$ allele shifted from a frequency of 0.359 at the lowest point on the transect $(1079 \mathrm{~m})$ to 0.927 at the highest point $(4054 \mathrm{~m})$. By contrast, frequencies of this same allele exhibited no systematic pattern of variation across the latitudinal transect (Figure 5C). None of the other loci exhibited a similar pattern of clinal variation across the altitudinal or latitudinal transects.

A weak pattern of isolation by distance was evident in samples of $P$. maniculatus from the altitudinal transect, as revealed by a positive association between pairwise $F_{\mathrm{ST}(\text { neutral) }}$ and pairwise geographic distance in both years (Figure 6A,B). By contrast, there was no evidence for an association between genetic differentiation and geographic distance across the latitudinal transect, which spanned a shorter linear distance (Figure 6C). If we restrict attention to the range of pairwise distances covered by the latitudinal transect (29.3-124.3 km), the association between neutral differentiation and geographic distance across the altitudinal transect is no longer significant $(P>0.05$ in both years). Moreover, weighted mean values of $F_{\mathrm{ST} \text { (neutral) }}$ were not significantly different between samples from the altitudinal and latitudinal transects over this same range of distances. Thus, there does not appear to be any substantial difference in the spatial scale of neutral differentiation across the altitudinal and latitudinal transects.

\section{Discussion}

Results of the coalescent simulations indicate that observed levels of divergence at the Alb locus reflect a history of diversifying selection across the altitudinal gradient. This conclusion supports the findings of a previous analysis that was based on a subset of the year 1 data (Storz and Nachman 2003), and is further bolstered by two main lines of evidence: (1) Alb exhibited a higher-than-expected level of divergence in two consecutive years of sampling across the altitudinal transect (in contrast to the spatial pattern of variation across the latitudinal transect) and (2) levels of divergence at the Alb locus were positively associated with altitudinal distance in both years (in contrast to the pattern observed at unlinked loci). Departures from neutral-

Table 2. Results of partial Mantel tests with restricted randomizations. The multiple regression model was designed to test for evidence of adaptive divergence across the altitudinal gradient. A matrix of $\arcsin \left(F_{\mathrm{ST}(A l b)}\right)^{1 / 2}$ was used as the dependent variable, and a matrix of arcsin $\left(F_{\mathrm{ST}(\text { neutral }}\right)^{1 / 2}$ was included as an independent variable to control for the effects of neutral genetic divergence. $P$-values are expressed as the proportion of 10,000 randomizations that yielded values greater than or equal to observed $t$ - or $F$-values.

\begin{tabular}{lllccc}
\hline & Regression coefficient & Estimate & $t^{1}$ & Extra SS & $F^{2}$ \\
\hline Year 1 & arcsin $\left(F_{\text {ST (neutral }}\right)^{1 / 2}$ & $b_{1}=2.804$ & $3.16(P<0.01)$ & 0.371 & $11.87(P<0.01)$ \\
& $\ln ($ altitudinal distance) & $b_{2}=0.042$ & $1.45(P<, 0.05)$ & 0.066 & $2.10(P<0.05)$ \\
Year 2 & $\arcsin \left(F_{\text {ST (neutral }}\right)^{1 / 2}$ & $b_{1}=1.892$ & $3.68(P<0.01)$ & 0.167 & $16.80(P<0.01)$ \\
& $\ln$ (altitudinal distance) & $b_{2}=0.052$ & $3.10(P<0.01)$ & 0.096 & $9.59(P<0.01)$ \\
\hline
\end{tabular}

\footnotetext{
${ }^{1}$ Associated $P$-values are for one-sided tests.

${ }^{2} \mathrm{~F}$-values are for the extra sum of squares accounted for by adding each variable into the equation.
} 

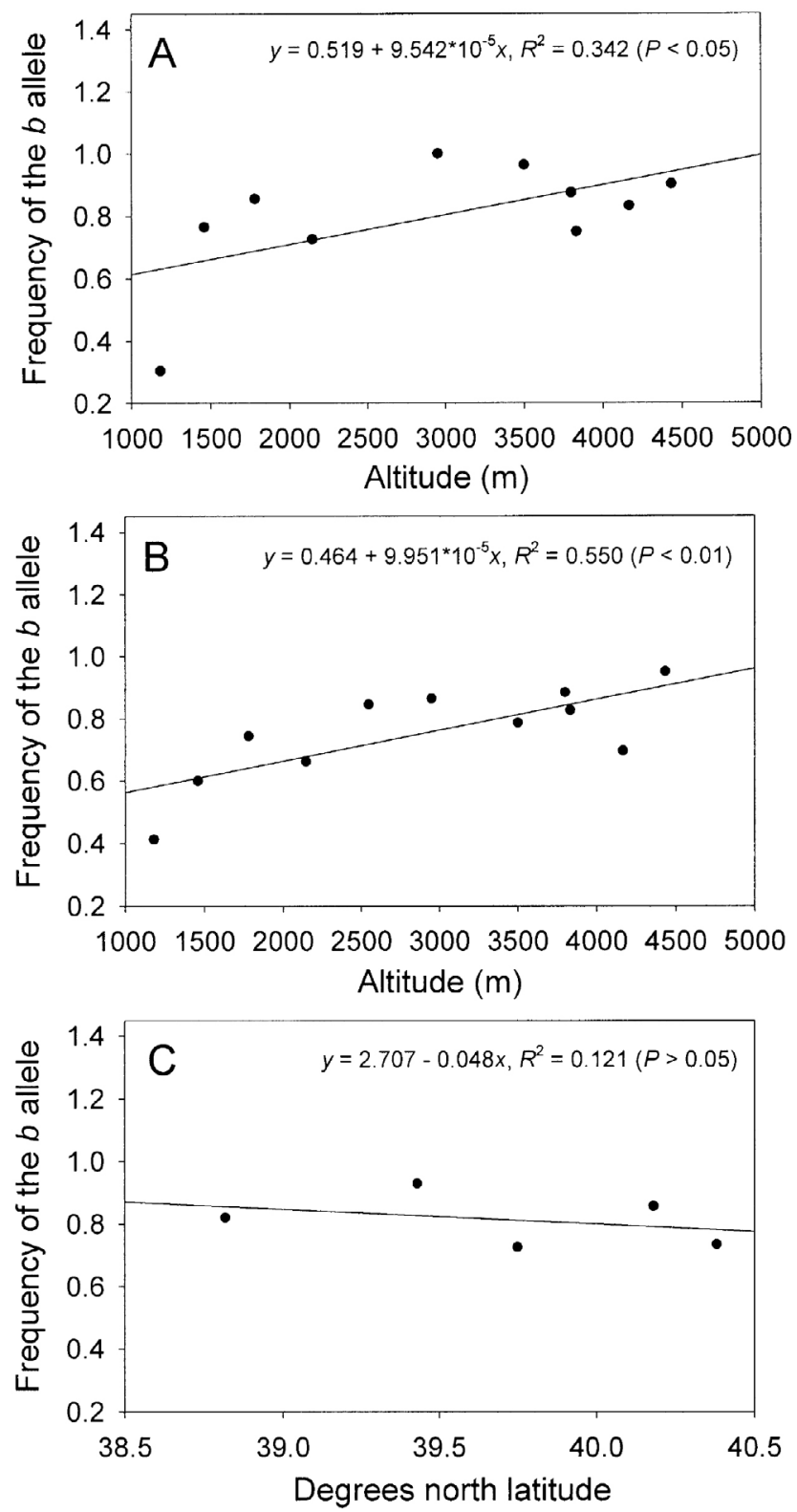

Figure 5. Linear regression of Alb allele frequency against altitude $(\mathrm{m})$ in (A) year 1 and (B) year 2, and regression of Alb allele frequency against latitude (C). $P$-values are one-tailed.

ity were highly significant in year 1 , and marginally significant in year 2 (after correcting for multiple comparisons), and the positive association between locus-specific divergence and altitudinal distance was highly significant in both years, even after controlling for the effects of neutral divergence. Taken together, these two lines of evidence strongly suggest that diversifying selection on the Alb locus is mediated by some environmental factor, such as ambient temperature or oxygen tension, that covaries with altitude.

The contrasting pattern of altitudinal divergence between Alb and the remaining loci indicates that diversifying selection has driven adaptive divergence in the face of extremely high levels of gene flow. All of the putatively neutral loci exhibited uniformly low $F_{\mathrm{ST}}$ values (Table 1). Using Wright's (1943) infinite-
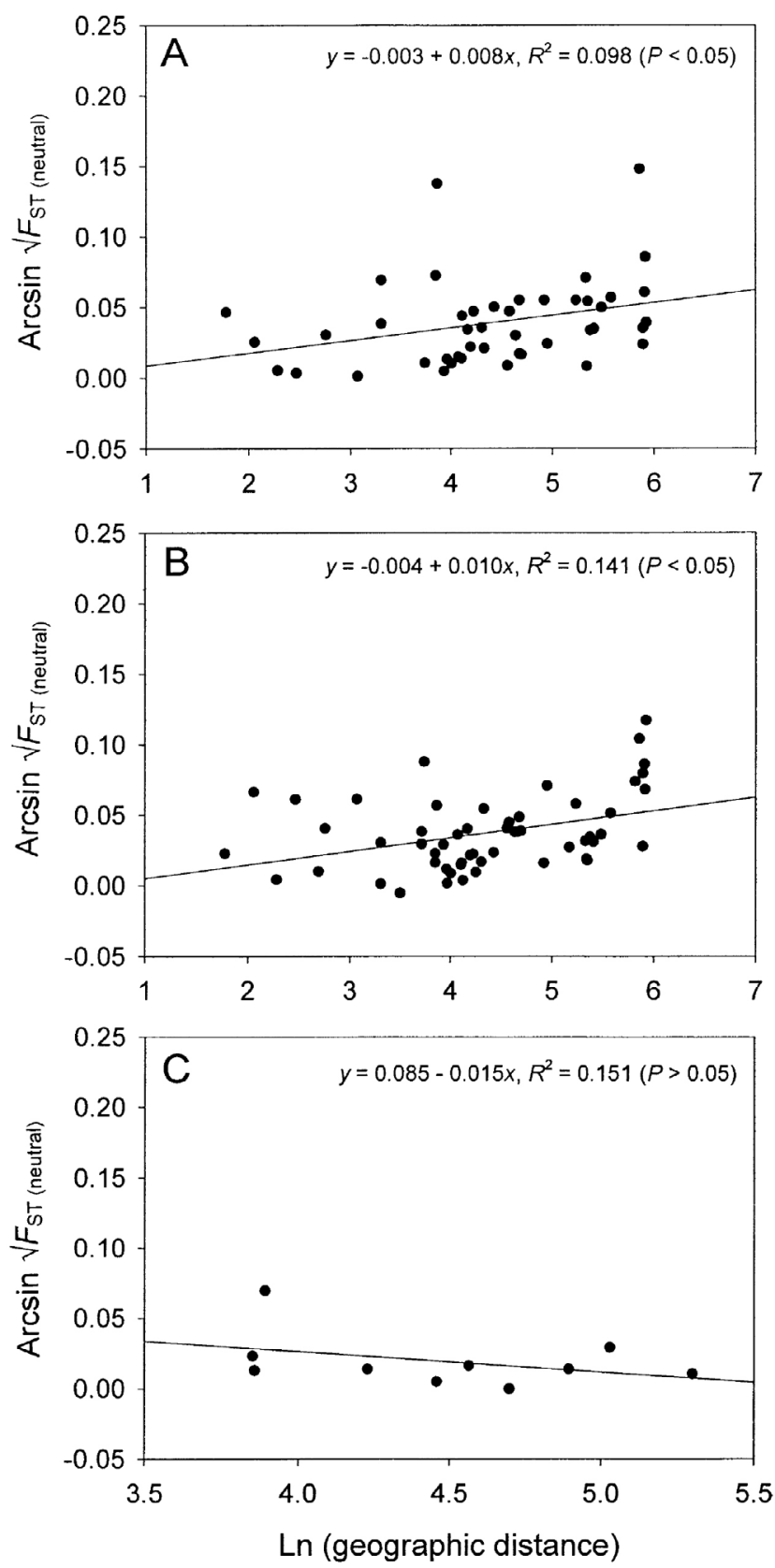

Figure 6. Linear regression of arcsin $\left(F_{\mathrm{ST}(\text { neutral }}\right)^{1 / 2}$ against ln (geographic distance) for each pairwise combination of sampling localities across the altitudinal transect (year 1, A; year 2, B) and across the latitudinal transect (C). $P$-values are from one-tailed randomization tests.

island approximation, $F_{\mathrm{ST}}=1 /\left(1+4 N_{e} m\right)$, the weighted mean values of $F_{\mathrm{ST} \text { (neutral) }}$ for year 1 (0.039) and year $2(0.038)$ translate into estimates of $N_{e} m \approx 6.16$ and 6.33 migrants/generation, respectively (where $N_{e} m$ is the migration rate scaled to local effective size). Kimura and Weiss (1964) showed that when $N_{e} m$ $>4$, the homogenizing effect of gene flow is sufficient to prevent stochastic differentiation of allele frequencies, such that the regional array of subpopulations is effectively panmictic. Under such conditions, local adaptation may be constrained by high levels of gene flow that produce a spatial averaging of fitness variation among different habitats (García-Ramos and Kirkpat- 
rick 1997; Lenormand 2002). We conclude that clinal variation at the Alb locus reflects a balance between gene flow and diversifying selection that results from elevational changes in fitness rankings among alternative genotypes.

All available evidence indicates that $P$. maniculatus is continuously distributed across the altitudinal gradient that we surveyed. With the exception of Alb, all loci exhibited a remarkably close fit to the expectations of the neutral model (Figure 2). If our transect had spanned a zone of secondary contact, the effects of admixture would have been readily detectable. Specifically, we would have seen systematic departures from Hardy-Weinberg genotypic proportions in addition to a bimodal distribution of pairwise $F_{\mathrm{ST}}$ values (corresponding to comparisons within and between samples from opposite sides of the contact zone). Admixture would also be expected to produce pervasive linkage disequilibria among loci that have different allele frequencies in the separate source populations. None of these patterns were evident. No cases of allelic or genotypic disequilibria remained significant after correcting for multiple comparisons. Moreover, the pattern of isolation by distance across the altitudinal gradient (Figure 6A,B) indicates that the regional population of $P$. maniculatus has attained migrationdrift equilibrium under extremely high levels of gene flow. The fact that all loci other than Alb conformed so closely to the null expectations indicates that the simulation model provides a reasonable approximation to the population structure of P. maniculatus over the spatial scale considered in this study.

In addition to the effects of selection on the distribution of Alb allele frequencies, patterns of variation will also be influenced by the same demographic processes that affect every other gene in the genome. Thus, positive associations between $F_{\mathrm{ST}(A l b)}$ and $F_{\mathrm{ST}(\text { neutral) }}$ in both years (Table 2) are not surprising given the high levels of gene flow.

In allozyme surveys of mice in the genus Peromyscus, Alb consistently emerges as one of the most highly differentiated loci, and statistically significant departures from neutral expectations have been documented in three other congeners of $P$. maniculatus (P. gossypinus, P. leucopus, and P. polionotus; Storz and Nachman 2003). It thus seems clear that Alb (or a closely linked locus) is subject to diversifying selection in multiple species of Peromyscus. However, since P. gossypinus, $P$. leucopus, and $P$. polionotus do not have very broad altitudinal distributions, the agent of selection in these species is probably not directly related to altitude.

The Alb data from the altitudinal and latitudinal transects illustrate how the same locus can exhibit both neutral and nonneutral patterns of variation depending on the environmental context. A similar situation has been documented for the glucose phosphate isomerase locus (Gpi) in populations of the northern acorn barnacle (Semibalanus balanoides) on the east coast of North America. Gpi genotypes in these barnacles are subject to fitness variation between high- and low-tide microhabitats in some coastal regions but not in others, possibly due to regional variation in the interaction between thermal stress and availability of dietary carbohydrates (Rand et al. 2002). In species like P. maniculatus that are distributed across ecologically heteroge- neous landscapes, allelic variation that is essentially neutral under most conditions may have hidden adaptive potential that is only exploited in certain environmental circumstances.

It is also possible that the apparent evidence for selection on Alb polymorphism is actually attributable to the indirect effects of selection at a closely linked locus. For example, a genetic study of European rat populations (Kohn et al. 2000) revealed that positive selection on $R w$, a gene that confers rodenticide resistance, induced a high degree of linkage disequilibrium across about $32 \mathrm{cM}(14 \%)$ of rat chromosome 1 . Thus, selection at a single locus may have affected patterns of variability at a multitude of linked sites within this chromosomal region (Kohn et al. 2000, 2003). Alb is a member of a multigene family consisting of three other genes that encode serum transport proteins: $\alpha$-fetoprotein, $\alpha$-albumin, and vitamin $\mathrm{D}$ binding protein. In rodents, the genes for albumin, $\alpha$-fetoprotein, and $\alpha$-albumin are closely linked within a $100-\mathrm{kb}$ block that is syntenic with the region centered on map position $50 \mathrm{cM}$ of Mus chromosome 5 . The gene for the vitamin D binding protein is situated further downstream at map position $44 \mathrm{cM}$ (Mouse Genome Informatics, available at http://www.informatics.jax.org). Even if the observed patterns of variability at the Alb locus reflect the indirect effects of selection at linked sites, closely linked members of the serum albumin gene family may be the most likely candidates for being the actual target of selection. If selection is sorting variation at a linked locus, then the expected level of differentiation at the unobserved polymorphism would be even higher than that observed at Alb, because the covariance in allele frequencies between the two loci would be dissipated at a rate proportional to the recombinational distance between them (Stephan and Mitchell 1992; Stephan 1994).

It is not clear what environmental factors may be mediating selection on the Alb locus in P. maniculatus. In mammals, albumin is a highly abundant serum protein that plays an important role in maintaining biochemical equilibria in the bloodstream and extravascular fluids. Albumin supplies most of the acid/ base buffering action of the plasma proteins (Figge et al. 1991) and it binds and transports a wide diversity of endogenous anions, particularly bilirubin, hematin, and long-chain fatty acids that are critical intermediates in lipid metabolism (Peters 1996). Albumin also binds nitric oxide (NO), an oxidizing gas with vasodilatory, antiplatelet, and neurotransmitting properties. Of the total NO in blood plasma, over $80 \%$ is carried as $S$-nitrosoalbumin (Keaney et al. 1993). The NO-binding properties of the protein suggest the possibility that high-altitude protein variants may mediate a vasodilatory response to hypoxic conditions. DNA sequence data may permit some inferences about functional differences among the allelic variants that predominate at different altitudes, but biochemical and/or physiological experiments will ultimately be required to determine the mechanistic basis of fitness differences among alternative Alb genotypes. By integrating surveys of geographic variation with detailed molecular-functional analyses, the study of allozyme variation can provide much insight into genetic mechanisms of physiological adaptation (Watt 1991; Mitton 1997; Eanes 1999; Watt and Dean 2000; Schmidt and Rand 2001). 


\section{Acknowledgments}

We thank J. Joule and M. Smith for helping with data collection, and J. Good, M. Goodisman, R. Harrison, E. Hebets, J. Mitton, M. Nachman, C. Roseman, and one anonymous reviewer for helpful comments on an earlier draft of this manuscript. We also thank A. Gibbs, D. Hahn, and T. Peters for helpful discussions of albumin biochemistry. JFS received support from a National Institutes of Health Postdoctoral Fellowship (F32 HL68487-01) and a Fellowship in Computational Molecular Biology from the Alfred P. Sloan Foundation and U.S. Department of Energy.

\section{Literature Cited}

Aquadro C. F., and J. C. Avise. 1982. An assessment of "hidden" heterogeneity within electromorphs at three enzyme loci in deer mice. Genetics. 102:269-284.

Baccus R., J. Joule, and W. J. Kimberling. 1980. Linkage and selection analysis of biochemical variants in Peromyscus maniculatus. J. Mammal. 61:423-435.

Beaumont M. A., and R. A. Nichols. 1996. Evaluating loci for use in the genetic analysis of population structure. Proc. R. Soc. Lond. B. 263:1619-1626.

Berry A., and M. Kreitman. 1993. Molecular analysis of an allozyme cline: alcohol dehydrogenase in Drosophila melanogaster on the east coast of North America. Genetics. 134:869-893.

Cavalli-Sforza L. L. 1966. Population structure and human evolution. Proc. R. Soc. Lond. B. 164:362-379.

Cockerham C. C., and B. S. Weir. 1993. Estimation of gene flow from $F$-statistics. Evolution. 47:855-863.

Dawson W. D. 1982. Protein polymorphisms in American deer mice (Peromyscus) and genetic linkage homology. Acta Theriol. 27:213-230.

Dawson W. D., L. L. Huang, M. R. Felder, and J. B. Shaffer. 1983. Linkage relationships among eleven biochemical loci in Peromyscus. Biochem. Genet. 21:1101-1114.

Duvernell D., P. S. Schmidt, and W. F. Eanes. 2003. Clines and adaptive evolution in the Methuselah gene region in Drosophila melanogaster. Mol. Ecol. 12:1277-1285.

Eanes W. F. 1999. Analysis of selection on enzyme polymorphisms. Annu. Rev. Ecol. Syst. 30:301-326.

Endler J. A. 1977. Geographic variation, speciation, and clines. Princeton Univ. Press, Princeton, NJ.

Endler J. A. 1986. Natural selection in the wild. Princeton Univ. Press, Princeton, NJ.

Figge J., T. H. Rossing, and V. Fencl. 1991. The role of serum proteins in acid-base equilibria. J. Lab. Clin. Med. 117:453-467.

García-Ramos G., and M. Kirkpatrick. 1997. Genetic models of adaptation and gene flow in peripheral populations. Evolution. 51:21-28.

Goudet J. 1995. FSTAT (Version 1.2): a computer program to calculate F-statistics. J. Hered. 86:485-486.
Haldane J. B. S. 1948. The theory of a cline. J. Genet. 48:277-284.

Keaney J. F. Jr., D. I. Simon, J. S. Stamler, O. Jaraki, J. Scharfstein, J. A. Vita, and J. Loscalzo. 1993. NO forms an adduct with serum albumin that has endothelium-derived relaxing factor-like properties. J. Clin. Invest. 91:1582-1589.

Kimura M., and J. F. Crow. 1964. The number of alleles that can be maintained in a finite population. Genetics. 49:725-738.

Kimura M., and G. H. Weiss. 1964. The stepping stone model of population structure and the decrease of genetic correlation with distance. Genetics. 49:561-576.

Kohn M. H., H.-J. Pelz, and R. K. Wayne. 2000. Natural selection mapping of the warfarin resistance gene. Proc. Natl. Acad. Sci. USA. 97:7911-7915.

Kohn M. H., H.-J. Pelz, and R. K. Wayne. 2003. Locus-specific genetic differentiation at $R w$ among warfarin-resistant rat (Rattus norvegicus) populations. Genetics. 164:1055-1070.

Lenormand T. 2002. Gene flow and the limits to natural selection. Trends Ecol. Evol. 17:183-189.

Lewontin R. C., and J. Krakauer. 1973. Distribution of gene frequency as a test of the theory of the selective neutrality of polymorphisms. Genetics. 74:175-195.

Manly F. J. B. 1997. Randomization, bootstrap and Monte Carlo methods in biology. Chapman and Hall, New York.

McDonald J. H. 1994. Detecting natural selection by comparing geographic variation in protein and DNA polymorphisms. Pp. 88100 in B. Golding, ed. Non-neutral evolution: theories and molecular data. Chapman and Hall, New York.

Mitton J. B. 1997. Selection in natural populations. Oxford Univ. Press, New York.

Nadeau J. H., and R. Baccus. 1981. Selection components of four allozymes in natural populations of Peromyscus maniculatus. J. Mammal. 61:423-435.

Nei M., and T. Maruyama. 1975. Lewontin-Krakauer test for neutral genes. Genetics. 80:395.

Oakeshott J. G., G. K. Chambers, J. B. Gibson, and D. A. Willcocks. 1981. Latitudinal relationships of esterase-6 and phosphoglucomutase gene frequencies in Drosophila melanogaster. Heredity. 47:385-396.

Oakeshott J. G., J. B. Gibson, P. R. Anderson, W. R. Knibb, D. G. Anderson, and G. K. Chambers. 1982. Alcohol dehydrogenase and glycerol-3-phosphate dehydrogenase clines in Drosophila melanogaster on different continents. Evolution. 36:86-96.

Ohta T., and M. Kimura. 1973. A model of mutation appropriate to estimate the number of electrophoretically detectable alleles in a finite population. Genet. Res. 22:201-204.

O'Farrell M. J. 1978. Home range dynamics of rodents in a sagebrush community. J. Mammal. 59:657-668.

Peters T. 1996. All about albumin: biochemistry, genetics, and medical applications. Academic Press, San Diego, CA.

Porter A. H. 2003. A test for deviation from island-model population structure. Mol. Ecol. 12:903-915.

Powers D. A., and A. R. Place. 1978. Biochemical genetics of Fundulus heteroclitus (L.). I. Temporal and spatial variation in gene frequencies of Ldh-B, Mdh-A, Gpi-B, and Pgm-A. Biochem. Genet. 16:593-607. 
Rand D. M, P. S. Spaeth, T. B. Sackton, and P. S. Schmidt. 2002. Ecological genetics of Mpi and Gpi polymorphisms in the acorn barnacle and the spatial scale of neutral and non-neutral variation. Integ. Comp. Biol. 42:825-836.

Robertson A. 1975a. Remarks on the Lewontin-Krakauer test. Genetics. 80:396.

Robertson A. 1975b. Gene frequency distributions as a test of selective neutrality. Genetics. 81:775-785.

Schmidt P. S., D. Duvernell, and W. F. Eanes. 2000. Adaptive evolution of a candidate gene for aging in Drosophila. Proc. Natl. Acad. Sci. USA. 97:10861-10865.

Schmidt P. S., and D. M. Rand. 2001. Adaptive maintenance of genetic polymorphism in an intertidal barnacle: habitat- and lifestage-specific survivorship of $\mathrm{Mpi}$ genotypes. Evolution. 55:1336-1344.

Selander R. K., M. H. Smith, S. Y. Yang, W. E. Johnson, and J. B. Gentry. 1971. Biochemical polymorphism and systematics in the genus Peromyscus. I. Variation in the old-field mouse (Peromyscus polionotus). Studies in genetics. VI. Univ. Texas. Publ. 7103:49-90.

Slatkin M. 1973. Gene flow and selection in a cline. Genetics. 75:733-756.

Sokal R. R., N. L. Oden, P. Legendre, M. Fortin, K. Junhyong, and A. Vaudor. 1989. Genetic differences among language families in Europe. Am. J. Phys. Anthropol. 76:337-361.

Sokal R. R., N. L. Oden, P. Legendre, M. Fortin, J. Kim, B. A. Thomson, A. Vaudor, R. M. Harding, and G. Barbujani. 1990. Genetics and language in European populations. Am. Nat. 135:157-175.

Stephan W. 1994. Effects of recombination and population subdivision on nucleotide sequence variation in Drosophila ananassae. Pp. 57-66 in B. Golding, ed. Non-neutral evolution: theories and molecular data. Chapman Hall, New York.
Stephan W., and S. J. Mitchell. 1992. Reduced levels of DNA polymorphism and fixed between-population differences in the centromeric region of Drosophila ananassae. Genetics. 132:1039-1045.

Storz J. F. 2002. Contrasting patterns of divergence between quantitative traits and neutral DNA markers: analysis of clinal variation. Mol. Ecol. 11:2537-2551.

Storz J. F., and M. W. Nachman. 2003. Natural selection on protein polymorphism in the rodent genus Peromyscus: evidence from interlocus contrasts. Evolution. 57:2628-2635.

Verrelli B. C., and W. F. Eanes. 2001. Clinal variation for amino acid polymorphisms at the Pgm locus in Drosophila melanogaster. Genetics. 156:1737-1752.

Vitalis R., K. Dawson, and P. Boursot. 2001. Interpretation of variation across marker loci as evidence of selection. Genetics. 158:1811-1823.

Watt W. B. 1991. Biochemistry, physiological ecology, and population genetics - the mechanistic tools of evolutionary biology. Funct. Ecol. 5:145-154.

Watt W. B., and A. M. Dean. 2000. Molecular-functional studies of adaptive genetic variation in prokaryotes and eukaryotes. Annu. Rev. Genet. 34:593-622.

Weir B. S., and C. C. Cockerham. 1984. Estimating F-statistics for the analysis of population structure. Evolution. 38:1358-1370.

Wright S. 1943. Isolation by distance. Genetics. 16:97-159. 